\title{
Phase transitions and gelation of silica-polystyrene mixtures in benzene
}

\author{
Gerrit A. Vliegenthart, ${ }^{* a b}$ Jeroen S. van Duijneveldt ${ }^{a}$ and Brian Vincent ${ }^{a}$ \\ ${ }^{a}$ School of Chemistry, University of Bristol, UK. E-mail: g.vliegenthart@fz-juelich.de. \\ ${ }^{b}$ Institut für Festkörperforschung, Forschungszentrum Jülich, Germany
}

Received 31st May 2002, Accepted 26th June 2002

First published as an Advance Article on the web 7th November 2002

\begin{abstract}
We have performed experiments on mixtures of sterically stabilised silica particles and large poly(styrene) polymers in benzene. Using this model system, we were able to independently vary short and long ranged attractive interactions by changing the temperature and/or the polymer concentration. We show that the addition of long ranged attractions to particles with initially only short ranged attractions leads to the appearance of a gas-liquid phase transition. Our results are in accordance with earlier theoretical predictions.
\end{abstract}

\section{Introduction}

Colloidal suspensions offer the unique opportunity to systematically study the role of attractive and repulsive interactions which determine the phase behaviour. The forces between particles in these suspensions can be tuned in strength and range by changing for instance solvent quality, ionic strength or addition of non-adsorbing polymer which induces depletion interactions. Long ranged attractive interactions in colloidal suspensions give rise to phase behaviour analogous to that of simple fluids involving a dense solid phase (colloidal crystal), a dense fluid (colloidal fluid) and a dilute fluid (colloidal gas) phase. In contrast to simple fluids, colloidal fluids often feature very short ranged attractive particle interactions. In that case a metastable gas-liquid phase transition is predicted but in only a few systems (solutions of proteins) is this transition actually observed (see for example refs. 1-5). In most cases the gas-liquid transition is preempted by gelation. Theoretically it has been predicted that the range of attractive interactions determines the location of the gel line with respect to the metastable gas-liquid curve. ${ }^{6-8}$

Schematically we summarise these predictions in Fig. 1 where we show as an example phase diagrams and the percolation line as a function of the temperature $T$ and the packing fraction $\eta$. For sufficiently short ranged interactions (Fig. 1(A)) there is no possibility for the system to phase separate, any quench in the part left of the percolation line leads initially to phase separation which is obstructed by gelation. Quenches right from the gel line lead to instantaneous and complete gelation of the sample. In Fig. 1(B) long ranged attractions have been increased and the gel line is shifted with respect to the gas-liquid phase boundary (or vice versa). A small temperature window where gas-liquid phase separation can occur has now appeared. Given this hypothesis it is therefore interesting to investigate the gelling properties of a model system in which the long and short ranged interactions can be characterised and varied independently. We report observations on such a model system to test these predictions and we discuss the interplay between phase separation and gelation as a function of temperature and polymer concentration.

DOI: $10.1039 / \mathrm{b} 205315 \mathrm{~b}$

Faraday Discuss., 2003, 123, 65-74 


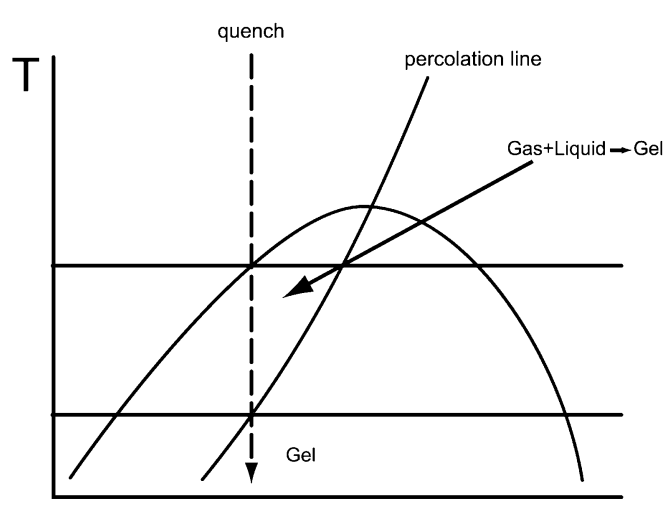

$\eta$

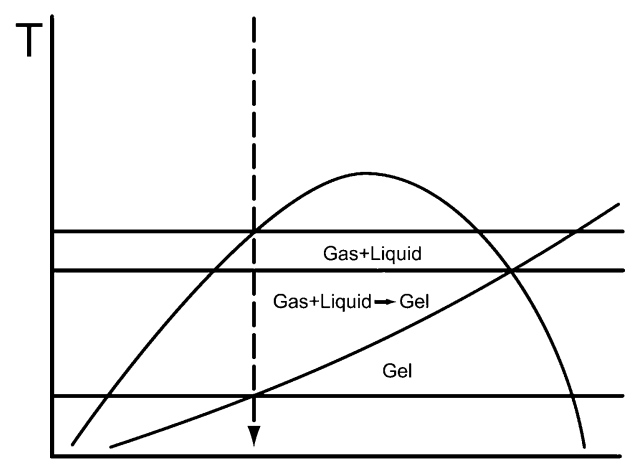

$\eta$

Fig. 1 Predicted schematic phase diagram for a system with mainly short (A), and combined short + long ranged attractions $(\mathrm{B})$.

Experiments have been performed on mixtures of stearyl coated silica particles and relatively large (non-adsorbing) poly(styrene) molecules in benzene. The shape of the interaction potential was systematically varied by changing the temperature and polymer concentration. In absence of free polymer, decreasing the temperature below a critical threshold value induces short ranged attractive interactions and the particles form a weak gel. The short ranged attractions are a consequence of the diminishing solvent quality for the stabilising hairs on the colloidal spheres with decreasing temperature and have in the past been modelled (successfully) using a polymer physics approach. ${ }^{9}$ If we consider the colloid-polymer mixture at high temperatures (where short ranged attractions are absent), we recover the well studied situation of silica particles experiencing a hard sphere repulsion plus a depletion induced attraction. For large polymer-to-colloid size ratios $q=2 R_{\mathrm{g}} / \sigma>0.3$ (where $R_{\mathrm{g}}$ is the radius of gyration of the polymer and $\sigma$ the diameter of the colloid) we expect the phase diagram to show a pronounced region of colloidal gas-liquid phase separation as is predicted by free volume theory ${ }^{10}$ and confirmed by experiments ${ }^{11,12}$ and computer simulations. $^{13,14}$

In our work we neglect the influence of van der Waals interactions between the silica particles as in earlier work of Jansen et al. ${ }^{15}$ indicates that these play only a minor role for this system. They calculated the Hamaker constant for stearyl coated silica particles in benzene to be approximately $0.6 k_{\mathrm{B}} T$, which is too small to cause thermodynamic instability.

In the following section we will give a brief description of the experimental model system. In section 3 the approximate interaction potentials for our model systems are discussed and we describe the application of a simple theoretical framework developed in ref. 6 to calculate the phase boundaries and the gel line. Finally in section 4, we present and discuss the results.

\section{Experimental methods}

\subsection{Preparation and characterisation of suspensions}

Silica particles were prepared using the Stöber method ${ }^{16}$ and subsequently grafted with stearyl alcohol. ${ }^{17}$ After removing excess stearyl alcohol by repeated centrifugation and redispersing in chloroform the particles were freeze dried and finally redispersed in benzene. The particle size was estimated using dynamic light scattering and transmission electron microscopy (TEM). For the hydrodynamic diameter we found $\sigma=112 \mathrm{~nm}$, while the TEM diameter of $100 \mathrm{~nm}$ was slightly lower. The polydispersity was obtained from the TEM images and found to be $15 \%$. Suspensions at 
room temperature $\left(25^{\circ} \mathrm{C}\right)$ were stable over weeks, but after a longer time some sedimentation was observed.

Monodisperse $\left(M_{\mathrm{w}} / M_{\mathrm{n}}=1.1\right)$ poly(styrene) of $2000 \mathrm{~kg} \mathrm{~mol}^{-1}$ was purchased from Polymer Source Inc. The polymer was dissolved by just leaving it overnight in a vial with benzene. The radius of gyration $R_{\mathrm{g}}$ of the polymer in benzene was measured using static light scattering and found to be $100 \mathrm{~nm}$. For such high molecular weights, extreme care has to be taken in handling the polymer as the polymers are extremely fragile and break up easily in shear flow.

\subsection{Colloid phase diagram}

The phase diagram of dispersions containing only the silica colloids (only short ranged interactions) was measured as a function of temperature by quenching samples of packing fractions $0<\eta<0.4$ in a water bath to the desired temperature. For inspection, the samples had to be taken out of the water bath and were then tilted/put upside down to observe signs of gelation. For a subset of samples we also studied the speckle pattern of the suspension which provides a less invasive method of investigating the samples.

\subsection{Colloid-polymer phase diagram}

The phase diagram at high temperature $\left(25^{\circ} \mathrm{C}\right.$, only long ranged interactions) was determined using the method of dilution lines. A number of colloid and polymer mixtures of different composition were prepared and on purpose, the initial polymer concentration was chosen to be high such that the mixtures were in the two phase region of the phase diagram and rapidly phase separating. The samples were then diluted with benzene until they became stable.

\subsection{Colloid-polymer phase diagram as a function of temperature}

For three packing fractions of colloid, $\eta=(\pi N / 6 V)=0.035,0.12$ and 0.2 , a number of polymer concentrations such that the mixtures were stable at $25^{\circ} \mathrm{C}$, the phase behaviour was examined as a function of temperature. Samples in 1 or $2 \mathrm{~mm}$ quartz cells (Hellma) were kept in a water bath at $25^{\circ} \mathrm{C}$ (stable conditions) and left for equilibration. The samples were then taken out and placed in a thermostated sample holder quenching the sample to the desired temperature. The temperature was measured using a reference cell in the same sample holder filled with pure solvent and a thermocouple. Although ideally the sample should be cooled homogeneously, this was not entirely possible in our experimental set up. The sample cell was cooled only from the sides. This was clearly visible in the increase of the turbidity with time where we observed 'dark' and more turbid layers growing from the sides of the cuvette which where cooled to the middle of the cell. The stability of the temperature control was about $0.3{ }^{\circ} \mathrm{C}$. After quenching a sample, the desired temperature was reached within $30 \mathrm{~s}$ for all cases.

A laser was shone through the sample and the speckle pattern was monitored over time (minutes to hours). Fluid phases always yielded vividly moving speckle patterns while the formation of a gel was indicated by slowing down or complete arrest of the speckle pattern. Furthermore, the viscosity of the samples in the final stage was probed crudely by tilting the samples or holding the samples upside-down. Samples that phase separated into a gas and liquid phase always had the characteristic mobile and sharp interface. Samples that gelled showed rigid (immobile when tilting) or rough interfaces and cracks in the bulk.

In our speckle experiment we measure the static percolation line as it is the point at which the speckles become static as a result of the particles becoming immobilised in a space spanning structure or in large flocs.

\section{Theoretical}

In order to test our assumptions on the interactions and phase behaviour of the experimental model system qualitatively, we have calculated the phase behaviour and percolation line using a simple theoretical approach. The model combines the equations of state from free volume theory ${ }^{10}$ for colloid-polymer mixtures (more correctly, mixtures of hard and penetrable hard spheres) and Baxter's adhesive hard sphere (AHS) model. ${ }^{18}$ The polymers are modelled as spheres with an 
equivalent diameter $\sigma_{\mathrm{p}}=2 R_{\mathrm{g}}$ which are fully penetrable for each other. The interactions between polymer spheres and colloidal spheres, and among colloidal spheres is hard sphere like. The equation of state (EOS) of the combined system is obtained following the procedure outlined in ref. 6 summing the attractive contributions due to short and long ranged attractions. We partially take into account the effect of the increased stickiness induced by long ranged attractions by using a renormalised stickiness parameter. This procedure gives the correct result on a second virial level. In subsection 3.1 we briefly introduce the free volume theory, after which we discuss the Baxter adhesive hard sphere model in subsection 3.2. In subsection 3.3 we describe the model for particles with both long and short ranged attractive interactions.

\subsection{Free volume equation of state}

The free volume equation of state for a mixture of hard spheres and penetrable hard spheres has been derived by Lekkerkerker et al. ${ }^{10}$ using the osmotic equilibrium formalism. In this formalism the free energy of the system is the sum of the free energy of the hard sphere reference fluid plus the free energy of the penetrable hard spheres in the available free volume. The free volume is obtained using scaled particle theory. ${ }^{19}$ Here we only give the final result for the equation of state,

$$
\frac{P v_{0}}{k_{\mathrm{B}} T}=P^{0}+\Pi\left(f-\eta \frac{\mathrm{d} f}{\mathrm{~d} \eta}\right)
$$

where $P^{0}$ is the pressure of the pure hard sphere system (i.e. the Percus-Yevick or the CarnahanStarling equation of state), $v_{0}$ the volume of a hard sphere, $k_{\mathrm{B}} T$ the thermal energy, $\Pi$ the osmotic pressure of the 'polymers' in the reservoir and proportional to the volume fraction of polymers $\eta_{\mathrm{p}}^{\mathrm{R}}$ in the reservoir, $f$ the fraction of the volume in the system available for the polymers and $\eta$ the packing fraction of hard spheres. Within the approximations of free volume theory $f$ is a function depending only on the polymer-to-colloid size ratio $q$ and the packing fraction of hard spheres. For small size ratios $(q<0.3)$ the free volume theory predicts the gas-liquid phase boundary to be metastable with respect to the fluid transition while for size ratios larger than 0.3 the gas-liquid transition is stable and becomes more and more pronounced. The critical point shifts to lower packing fractions while the critical polymer concentration shifts to higher values with increasing size ratio. These trends have been confirmed by computer simulations. ${ }^{14}$

\subsection{Adhesive hard sphere model}

The Baxter adhesive hard sphere model has in the past been successfully applied to characterise the interactions and explain the phase behaviour of sticky colloids. ${ }^{15}$ The interaction potential in this model is given by

$$
V(r)=\lim d \rightarrow \sigma \begin{cases}\infty & r<\sigma \\ k_{\mathrm{B}} T \ln 12 \tau\left[\frac{d-\sigma}{\sigma}\right] & \sigma<r<d, \\ 0 & r>d\end{cases}
$$

Here $\tau$ is the 'stickiness' parameter and $d-\sigma$ the width of the potential well. For this potential, the Percus-Yevick equation for the pair correlation function can be solved exactly and the thermodynamic properties of the system can be calculated via the pressure, compressibility or energy route. The equation of state resulting from the compressibility route equals

$$
\frac{P v_{0}}{k_{\mathrm{B}} T}=\eta \frac{\left(1+\eta+\eta^{2}\right)}{(1-\eta)^{3}}-\frac{36 \eta \lambda(1+\eta / 2)+\lambda^{3}}{36 \eta(1-\eta)^{3}}
$$

where $\lambda$ is a function of the stickiness and the density (see, for the actual expression, ref. 18). The critical point for this equation of state is located at $\eta=0.12$ and $\tau=0.0976$. Besides thermodynamic quantities, structural information can be obtained in the form of the percolation line, i.e. the line at which the average cluster size diverges, ${ }^{20}$

$$
\tau \leq \frac{1-2 \eta+19 \eta^{2}}{12(1-\eta)^{2}}
$$


The percolation line from this theory is not by definition the same as the one we measure in our experiment but it is in this case the point at which the system forms space spanning clusters, while these clusters can be dynamic. In the experiments we measure the static percolation line.

To connect with experiments, the adhesive hard sphere potential is mapped on a continuous short ranged potential via the second virial coefficient. In this way the Baxter stickiness parameter $\tau$ is related to a real temperature,

$$
B_{2}=v_{0}\left(1-\frac{1}{4 \tau}\right)=2 \pi \int_{0}^{\infty}\left(1-\exp \left[-V(r) / k_{\mathrm{B}} T\right]\right) r^{2} \mathrm{~d} r
$$

The width of this potential $V(r)$ should be small compared to the particle size, of the order of the length of the chains on the surface and is somewhat arbitrary.

In our case we take for the short ranged potential,

$$
V(r)=\varepsilon(T)\left(\frac{\sigma}{r}\right)^{200}
$$

where $\varepsilon$ is a temperature dependent interaction strength,

$$
\varepsilon=-L\left(\frac{\theta}{T}-1\right) k_{\mathrm{B}} T
$$

with $L$ proportional to the overlapping volume of chains on both particles and $\theta$ the temperature at which the attraction vanishes. The values for $L$ and $\theta$ were taken from ref. 21 .

\subsection{The free volume equation of state including short ranged adhesive interactions}

In the case of summation of a long and an infinitely short ranged attraction, both will contribute to the contact value of the potential and it is easily shown that on a second virial level $\tau$ is renormalised by a factor $\mathrm{e}^{W(0) / k_{\mathrm{B}} T}$. For example, in case of summation of the sticky sphere potential and a square well potential with range $\gamma$ and depth $\varepsilon$, the second virial coefficent is given by,

$$
B_{2}=B_{2}^{\mathrm{HS}}\left(1-\frac{\mathrm{e}^{\varepsilon / k_{\mathrm{B}} T}}{4 \tau}\right)+B_{2}^{\mathrm{HS}}\left(\gamma^{3}-1\right)\left(1-\mathrm{e}^{\varepsilon / k_{\mathrm{B}} T}\right)
$$

which contains the hard sphere repulsive part $B_{2}^{\mathrm{HS}}=(2 \pi / 3) \sigma^{3}$, the renormalised short ranged attractions and the square-well attractive part. In case of long ranged depletion interactions we use for $\varepsilon$ just the depletion potential at contact,

$$
\frac{W(0)}{k_{\mathrm{B}} T}=-\frac{1}{q}[1.5+q] \eta_{\mathrm{p}}^{\mathrm{R}}
$$

In our model for the EOS of the system with both short and long ranged attractions we just sum the two attractive contributions and use for $\tau$ the renormalised value $\bar{\tau}=\tau / \mathrm{e}^{W(0) / k_{\mathrm{B}} T}$.

The gas-liquid branch of the EOS is now given by,

$$
\frac{P v_{0}}{k_{\mathrm{B}} T}=\eta \frac{\left(1+\eta+\eta^{2}\right)}{(1-\eta)^{3}}-\frac{36 \eta \bar{\lambda}\left(1+\frac{\eta}{2}\right)+\bar{\lambda}^{3}}{36 \eta(1-\eta)^{3}}-\frac{\eta_{\mathrm{p}}^{\mathrm{R}}}{q^{3}}\left(f-\eta \frac{\mathrm{d} f}{\mathrm{~d} \eta}\right)
$$

Here $\eta_{\mathrm{p}}^{\mathrm{R}}=(4 \pi / 3) R_{\mathrm{g}}^{3}$ is the polymer volume fraction in the osmotic reservoir and $\bar{\lambda}=\lambda(\bar{\tau}, \eta)$.

The first term on the rhs of eqn. (10) is the Percus-Yevick hard sphere pressure, the second term takes into account the short ranged sticky interactions (following Baxter) and the last term results from the depletion attractions. The chemical potential is obtained using the Gibbs-Duhem relation and given the rather lengthy expression we refer to ref. 22 for the AHS contribution and to ref. 10 for the depletion contribution. The binodal is constructed by requiring equality of pressure and chemical potential of both phases. The percolation line is calculated using eqn. (4) using the renormalised value $\bar{\tau}$.

The procedure is thus as follows; for a given value of (the bare) $\tau$ and $\eta_{\mathrm{p}}^{\mathrm{R}}$ calculate the corresponding temperature via $B_{2}$, then calculate $\bar{\tau}$ and finally calculate the phase boundary and the percolation line using $\bar{\tau}$. 


\section{Results and discussion}

\subsection{General remarks}

As the refractive indices of silica (1.45) and benzene (1.5) are not too similar, the turbidity of the samples is significant, even for low volume fractions. This clearly makes it difficult to see the interfaces by eye. The polydispersity of the spheres prevents them from crystallising.

\subsection{Colloid phase diagram as function of temperature}

Fig. 2 shows the phase diagram of the pure colloid as a function of temperature. All samples become unstable or gel completely at temperatures around $12-13{ }^{\circ} \mathrm{C}$. for high packing fractions $(\eta>0.2)$, the samples completely gel upon cooling. For the lower packing fractions $(\eta<0.2)$ there is a small temperature window in which the suspensions start to phase separate (indicated by the appearance of a sharp and initially mobile meniscus coming from the top, in the sample tube) but in this case the samples get 'stuck' in a gel state in the process of phase separation. The phase separation is observed quite rapidly, typically after $5-15 \mathrm{~min}$. The gel state is reached after $30 \mathrm{~min}$. At low packing fractions, a temperature where the suspension completely gels is absent (as it is probably below temperatures where the solvent freezes). In all cases the gels formed are very weak, having a low yield stress. Keeping a sample tilted leads to flow of the gel-air or gel-fluid interface within seconds and subsequently the gel fully broke up to give a liquid-liquid (non-equilibrium) 'coexistence'. For the lowest volume fractions we observed the appearance of a dense amorphous phase 'growing' from the bottom of the tube. The nature of this phase is unclear but it appears to be a dense fluid slowly being transformed into a solid phase. Tilting the cuvette induced a kind of 'shear melting' of the top of this phase which looks different from either a gelled or a fluid sample being tilted. For more concentrated samples we did not observe this behaviour but that could easily be due to the high turbidity of the samples. We note that in our experimental system the temperatures at which the instability occurs is approximately 5 degrees lower than in a similar system (stearyl coated silica of $100 \mathrm{~nm}$ diameter in benzene) explored by Verduin and Dhont, ${ }^{21}$ and appears to be much lower than in systems equivalent to those studied by Rouw and de Kruif ${ }^{23}$ and Jansen et al. ${ }^{15}$ This is most likely due to a different grafting density of stearyl alcohol chains on the silica particles which influences both the entropic and enthalpic contribution of the attraction between the chains.

We note we have found only one reported observation in literature (as we know) of a liquidliquid-like phase separation of a stearyl silica in benzene, ${ }^{15}$ in all other cases samples showed gelation or partial phase separation followed by gelation.

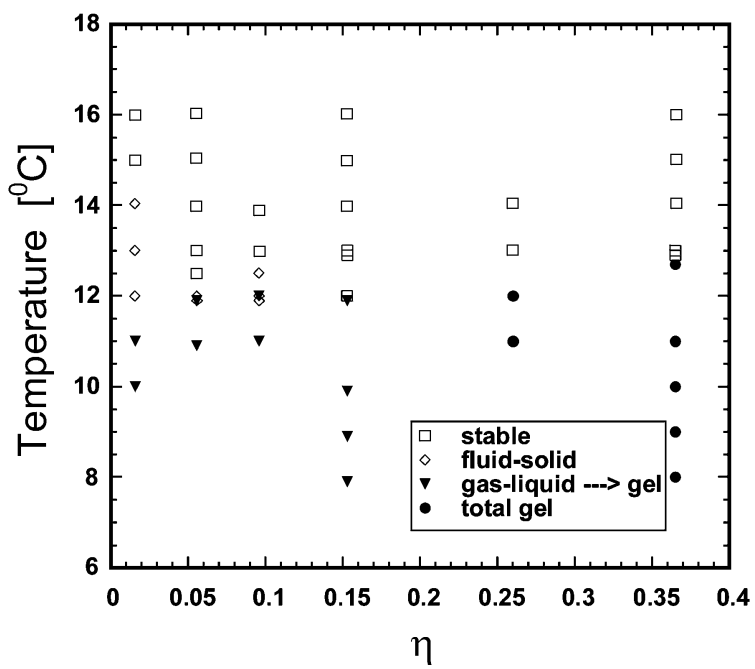

Fig. 2 The experimental phase diagram of the pure stearyl silica in benzene as a function of temperature. 


\subsection{Colloid-polymer phase diagram at $25^{\circ} \mathrm{C}$}

In Fig. 3(A), the gas-liquid phase diagram of the colloid-polymer mixture is given. Note that the volume fractions of polymer $\eta=(4 N) /(3 V) R_{\mathrm{g}}^{3}$ required for phase separation are quite large. In Fig. 3 (B) we show the corresponding free volume result for $q=2.0$ (taking the radius of the penetrable hard sphere equal to the radius of gyration of the polymer) which clearly overestimates the strength of the depletion interactions compared to the experiments. Given the severe approximations of free volume theory which replaces real polymers by penetrable hard spheres and thus ignores the internal structure of the polymer and which does not take into account many body depletion interactions which may play a role for $q>0.154$ the results are surprisingly good. Recent extensions of the original free volume theory, ${ }^{24}$ taking into account a concentration-dependent depletion layer thickness and excluded volume interactions between the polymers show a much better agreement between theoretical and experimental phase diagrams. We note that for the gas-liquid phase separation process, a diffuse meniscus first appears in the bulk of the sample. The exact location depends on the colloid and polymer concentrations in the sample. This is a different scenario compared to samples which finally gel, where we observe a meniscus coming from the top.

\subsection{Colloid-polymer mixture as a function of temperature}

Apart from a depletion-induced phase transition and a temperature-induced gel transition, two other possible mechanisms for phase separation and gelation are to be eliminated first. We first checked for phase separation of the polymer itself, by cooling a concentrated solution of poly(styrene) to different temperatures. In none of the cases we found evidence for such a phase separation. Secondly, the polymer could induce a gel transition as has been frequently observed and studied for small polymer to colloid size ratios. ${ }^{25}$ It appeared that also for large polymer to colloid size ratios a state of high viscosity (gel) can be induced but this situation arises at extremely high polymer concentrations $\left(C_{\mathrm{p}}=0.01 \mathrm{~g} \mathrm{ml}^{-1}\right)$, clearly much higher than used in our experiments. The aggregation/gelation for systems with long ranged interactions has also been observed in computer simulations of Lennard-Jones spheres ${ }^{26}$ quenched to very low temperatures, typically deep under the triple point of the Lennard-Jones system.

In Fig. 4 we show the phase behaviour for colloid-polymer mixtures as a function of temperature. Firstly, all samples are stable at $25^{\circ} \mathrm{C}$ (indicated by open squares), so in the one-phase region of the colloid-polymer mixture. Upon cooling, short ranged attractions are increased are and the samples can become unstable. According to Fig. 1 the following scenarios are then possible,

(i) undergoing 'proper' gas-liquid phase separation (filled upright triangles)

(ii) initially starting to gas-liquid phase separate, then the phase concentrated in colloid gets stuck in a gel (filled downward triangles),

(iii) gelling fully (filled circles).
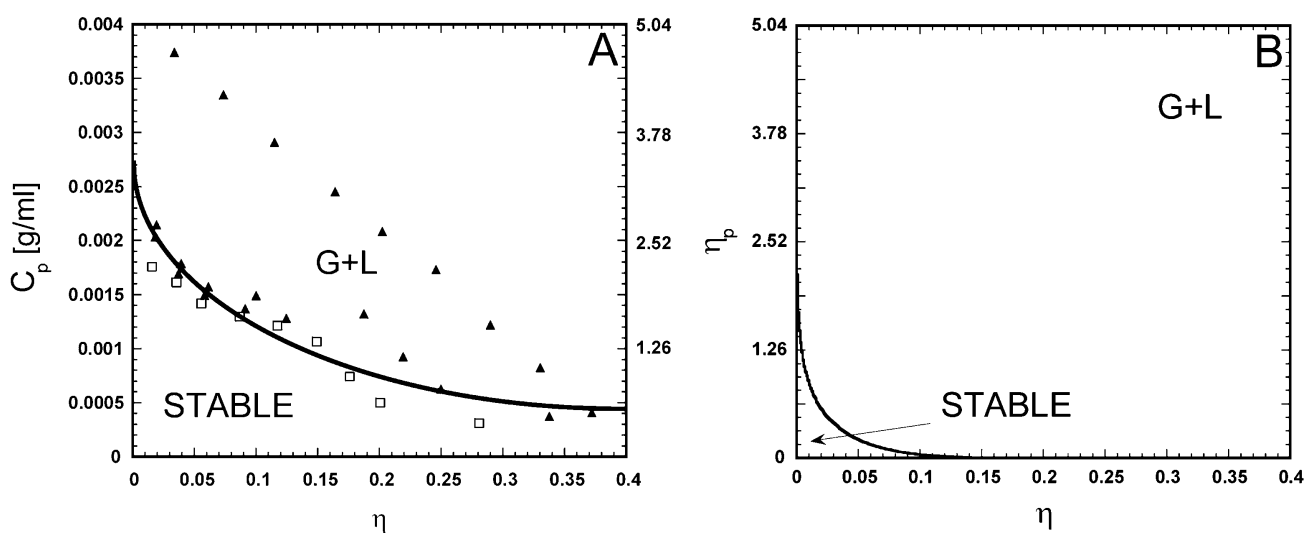

Fig. 3 (A) The experimental phase diagram of the colloid-polymer mixture, (B) Free volume result. 

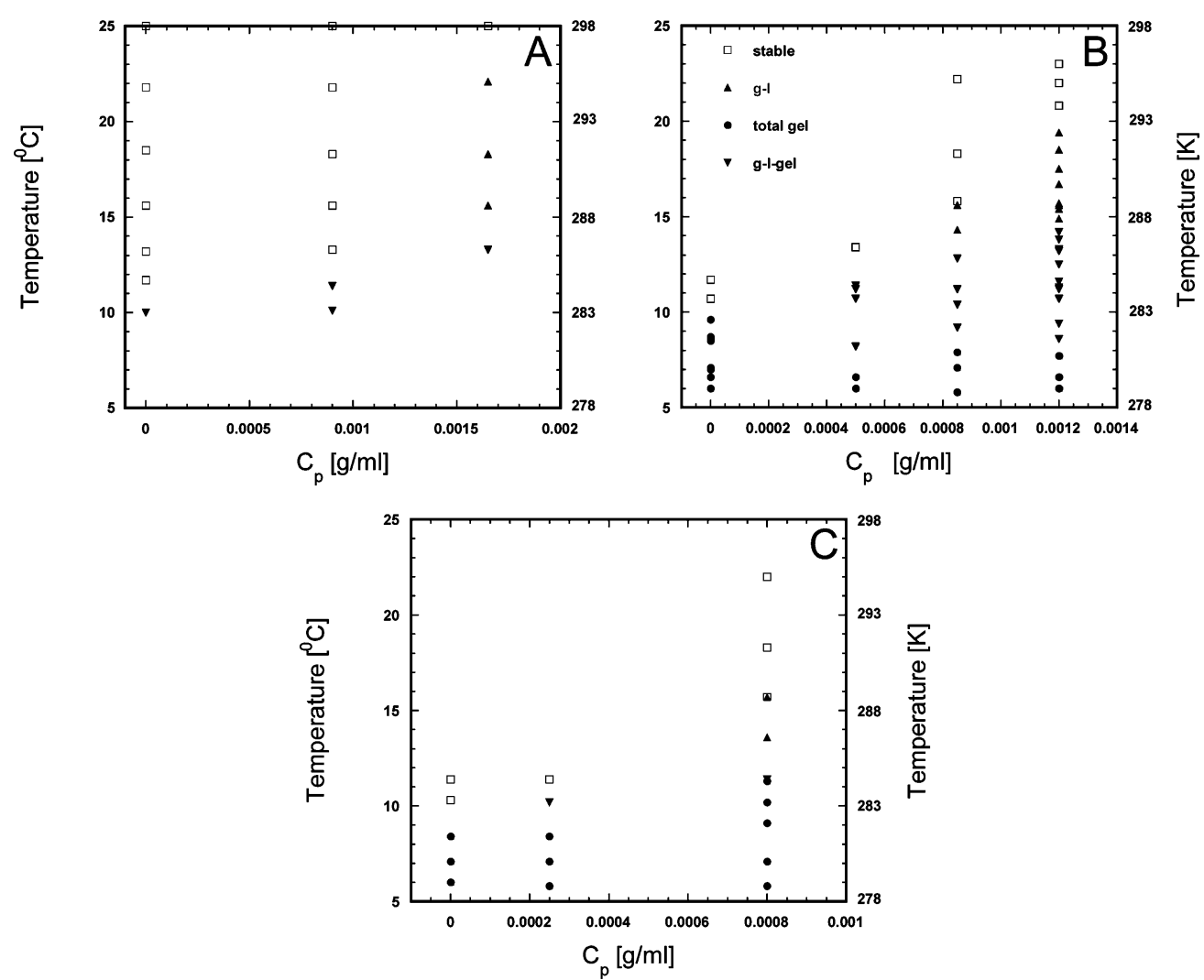

Fig. 4 The phase diagram of the colloid-polymer mixture as a function of temperature, (A) $\eta_{\mathrm{c}}=0.035$, (B) $\eta_{\mathrm{c}}=0.12$, (C) $\eta_{\mathrm{c}}=0.2$.

Fig. 4(A) shows the phase behaviour of a suspension of $\eta=0.035$ for three different polymer concentrations. If no polymer is added, we find a transition from the stable to the unstable regime at $11^{\circ} \mathrm{C}$. For larger polymer concentrations, this transition shifts to slightly higher temperatures thus shifting the gel line. For the highest polymer concentration we find a gas-liquid transition between 15 and $22^{\circ} \mathrm{C}$. For this sample of low colloid packing fraction we did not observe the fluidsolid transition indicated in the previous section.

In Fig. 4(B) we show the results for a suspension of $\eta=0.12$. In absence of polymer, we find that the dispersion completely gels below 10 and $11^{\circ} \mathrm{C}$ above which it is stable. For slightly higher polymer concentrations, we first see the appearance of a gas-liquid phase separation getting stuck in a gel while for higher polymer concentrations a stable gas-liquid phase transition appears.

For the highest packing fraction $\eta=0.2$ which is displayed in Fig. 4(C), we observe the same trend. For low polymer concentrations the system goes directly from stable to fully gelled, while for the intermediate polymer concentrations, an attempt to gas-liquid phase separation is made and for the intermediate and highest polymer concentrations the system exhibits a region of gas-liquid phase transition broadening with increasing polymer concentrations.

We note that the location of the gel line (crossover from filled circles to filled downward triangles or filled circles to open squares) changes with polymer concentration but not a great deal. Interestingly, for $\eta=0.12$, the gel line seems to be slightly suppressed at intermediate polymer concentrations.

In Fig. 5 we show the sample at $\eta=0.12$ and $C_{\mathrm{p}}=0.0012 \mathrm{~g} / \mathrm{ml}$ which has been quenched from $25^{\circ} \mathrm{C}$ to $8.6^{\circ} \mathrm{C}$ and then heated up to $17^{\circ} \mathrm{C}$. The cooling from 25 to $8.6^{\circ} \mathrm{C}$ results in the formation of a gel. Within minutes the speckle pattern froze, after which the gel started to age. Vertical cracks 


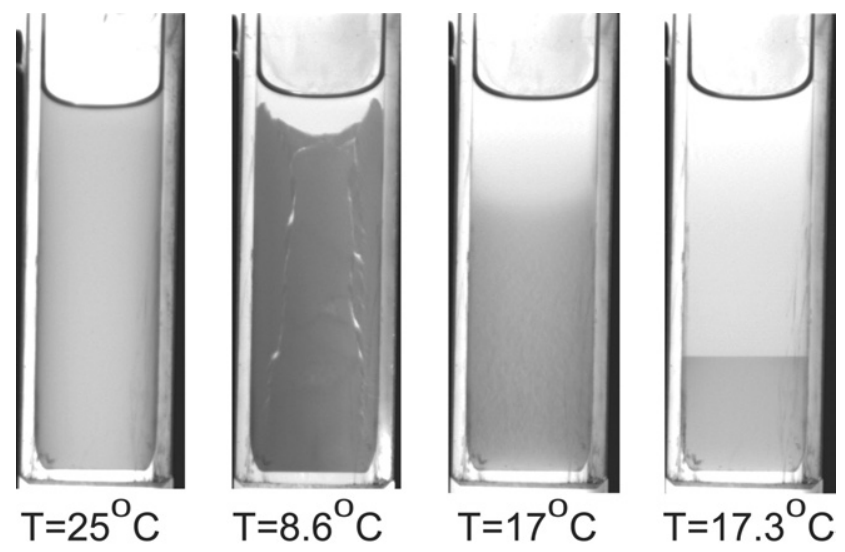

Fig. 5 Colloid polymer mixture of $\eta=0.12$ and $C_{\mathrm{p}}=0.0012 \mathrm{~g} \mathrm{ml}^{-1}$.

appeared near the walls which were cooled, and the gel-solvent interface showed characteristic 'owls ears' on top. This morphology closely resembles that found by Weeks et al. ${ }^{27}$ in cooling experiments of silica particles grafted with long poly(styrene) molecules in cyclohexane. Subsequently, the gelled sample was heated up setting the thermostat to $17^{\circ} \mathrm{C}$ (a temperature of which we knew in advance that the sample would tend to do a gas-liquid phase separation) and while heating (and thus turning of short ranged interactions) the gel slowly changed appearance, the ears fell down, cracks were filled up, and finally the system phase separated into a coexisting gas and liquid.

Fig. 6 shows the gas-liquid phase boundary and the percolation line as a function of temperature for two polymer concentrations, $\eta_{\mathrm{p}}^{\mathrm{R}}=0.45$ (open symbols) and $\eta_{\mathrm{p}}^{\mathrm{R}}=0.9$ (filled symbols) calculated using the theoretical approach outlined in section 3. In Fig. 6(A) we show the phase boundaries and percolation line for the case that the stickiness is not renormalised by the long ranged interactions. The percolation line is then the same for both polymer concentrations, but increasing the polymer concentration leads to a significant rise of the critical temperature and thus there is a wider temperature window for gas-liquid phase separation. If the contact value of the depletion interactions
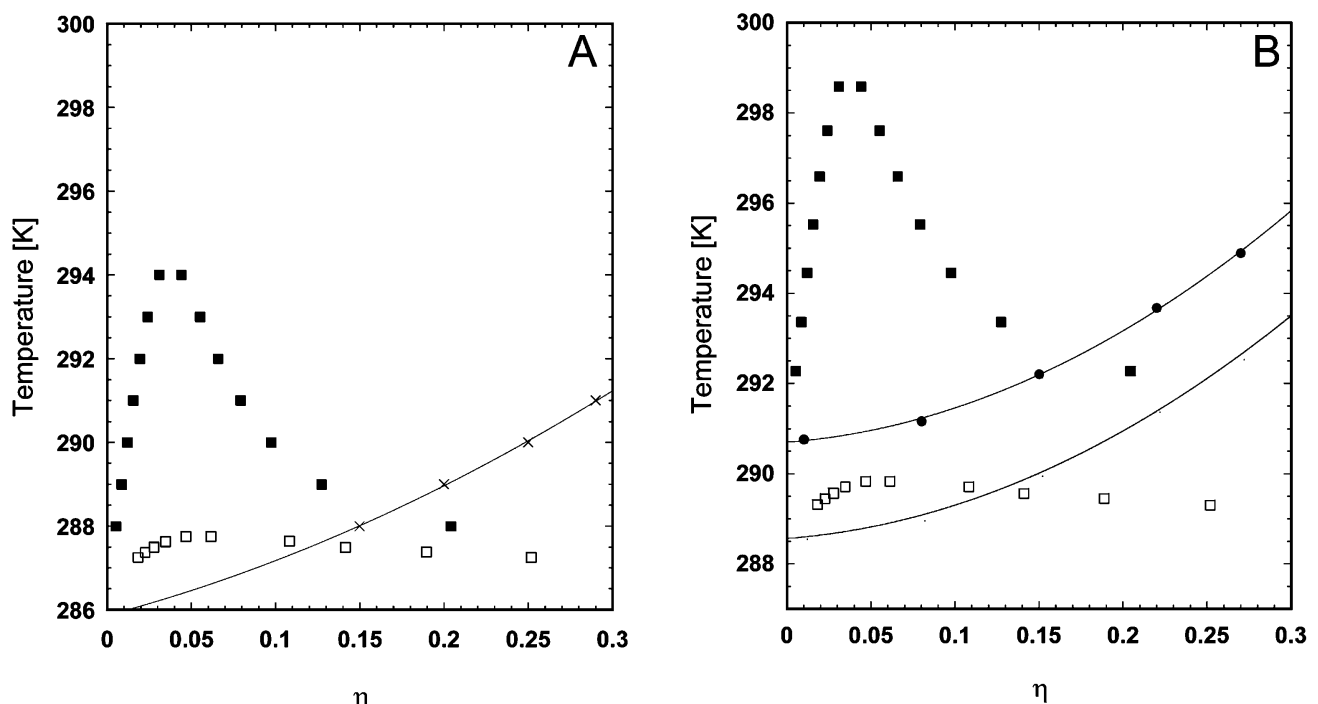

Fig. 6 Theoretical phase diagrams (open and filled squares) and percolation lines (drawn lines) for $\eta_{\mathrm{p}}^{\mathrm{R}}=0.45$ (open symbols) and $\eta_{\mathrm{p}}^{\mathrm{R}}=0.9$ (filled symbols). (A) $\tau$ not renormalised, (B) $\tau$ renormalised. 
is taken into account in the stickiness, the picture changes and both the percolation line and the phase boundary shift when changing the polymer concentration. This is illustrated in Fig. 6. Also in this case, increasing the polymer concentration enlarges the possibilities for gas-liquid phase separation. There is however no qualitatively different behaviour. This result is in qualitative agreement with that from [6] and supports our experimental result.

Summarising, for all samples without polymer, a region of gas-liquid phase separation is absent. For samples close to the depletion binodal, a proper gas-liquid phase transition is observed. The extra attraction due to the sticky interactions is sufficient to drive the system over the phase boundary but not sufficient to cause the particles to cluster irreversibly. The location of the gel line is only slightly dependent on the addition of long ranged attractions and can be either shifted to lower or to higher temperatures depending on the exact location in the phase diagram. The theory predicts the gel line to be shifted to higher temperatures with increasing long ranged attractions.

Protein solutions form a class of colloidal suspensions for which short ranged attractions are assumed and for which the meta-stable gas-liquid phase transition has really been observed. In that case long ranged van der Waals interactions, dipolar interactions or specific interactions might be just strong enough to shift the percolation line such that there is a slight opportunity for gas-liquid phase separation.

\section{Acknowledgements}

This work was funded by EU Grant No. MCFI-1999-00115 [GV]. We acknowledge support from the Engineering and Physical Sciences Research Council for grants towards computer hardware (GR/M34799,GR/N15696). We thank Bob Evans, Henk Lekkerkerker, Paul van der Schoot, Jan Dhont and Remco Tuinier for useful discussions. GV thanks the School of Chemistry at the University of Bristol for its hospitality!

\section{References}

1 C. Ishimoto and T. Tanaka, Phys. Rev. Lett., 1977, 39, 474-477.

2 G. Phillies, Phys. Rev. Lett., 1985, 55, 1341-1341.

3 M. Broide, C. Berland, J. Pande, O. Ogun and G. Benedek, Proc. Natl. Acad. Sci. USA, 1991, 88, 5660 5664.

4 C. Berland, G. Thurston, M. Kondo, M. Broide, J. Pande, O. Ogun and G. Benedek, Proc. Natl. Acad. Sci. USA, 1992, 89, 1214-1218.

5 M. Muschol and F. Rosenberger, J. Chem. Phys., 1997, 107, 1953-1962.

6 M. Noro, N. Kern and D. Frenkel, Europhys. Lett., 1999, 48, 332-338.

7 J. Bergenholtz and M. Fuchs, J. Phys.: Condens. Matter, 1999, 11, $10171-10182$.

8 J. Bergenholtz and M. Fuchs, Phys. Rev. E, 1999, 59, 5706-5715.

9 P. Flory and W. Kriegbaum, J. Chem. Phys., 1950, 18, 1086-1094.

10 H. Lekkerkerker, W. Poon, P. Pusey, A. Stroobants and P. Warren, Europhys. Lett., 1992, 20, 559-564.

11 C. Cowell, R. Lin-In-On and B. Vincent, J. Chem. Soc., Faraday Trans. 1, 1978, 74, 337-347.

12 A. V. H. de Hek, J. Colloid Interface Sci., 1981, 84, 409-422.

13 E. Meijer and D. Frenkel, J. Chem. Phys., 1994, 100, 6873-6887.

14 M. Dijkstra, J. Brader and R. Evans, J. Phys.: Condens. Matter, 1999, 11, $10079-10106$.

15 J. Jansen, C. de Kruif and A. Vrij, J. Colloid Interface Sci., 1986, 114, 471-480.

16 W. Stöber, A. Fink and E. Bohn, J. Colloid Interface Sci., 1968, 26, 62-69.

17 A. van Helden, J. Jansen and A. Vrij, J. Colloid Interface Sci., 1981, 81, 354-367.

18 R. Baxter, J. Chem. Phys., 1968, 49, 2770-2774.

19 H. Reiss, H. Frisch and J. Lebowitz, J. Chem. Phys., 1959, 31, 369-380.

20 Y. Chiew and E. Glandt, J. Phys. A, 1983, 16, 2599-2608.

21 H. Verduin and J. Dhont, J. Colloid Interface Sci., 1995, 172, 425-437.

22 C. Tejero and M. Baus, Phys. Rev. E, 1994, 48, 3793-3798.

23 P. Rouw and C. de Kruif, Prog. Colloid Polym. Sci., 1988, 76, 1-15.

24 D. G. A. L. Aarts, R. Tuinier and H. N. W. Lekkerkerker, J. Phys.: Condens. Matter, 2002, 14(33), $7551-7561$.

25 W. Poon, A. Pirie and P. Pusey, Faraday Discuss., 1995, 101, 65-76.

26 J. Lodge and D. Heyes, J. Chem. Soc., Faraday Trans I, 1997, 93, 437-448.

27 J. Weeks, J. van Duijneveldt and B. Vincent, J. Phys.: Condens. Matter, 2000, 12, 9599-9606. 